Last Update: 09 June 1995

hep-th/9502075

\title{
A Massive Renormalizable Abelian Gauge Theory in 2+1 Dimensions
}

\author{
F.A. Dilkes \\ D.G.C. McKeon \\ Department of Applied Mathematics \\ University of Western Ontario \\ London CANADA \\ N6A 5B7
}

email: TMLEAFS@APMATHS.UWO.CA

PACS No.: 11.10.Gh 


\section{Abstract}

The standard formulation of a massive Abelian vector field in $2+1$ dimensions involves a Maxwell kinetic term plus a Chern-Simons mass term; in its place we consider a ChernSimons kinetic term plus a Stuekelberg mass term. In this latter model, we still have a massive vector field, but now the interaction with a charged spinor field is renormalizable (as opposed to super renormalizable). By choosing an appropriate gauge fixing term, the Stuekelberg auxiliary scalar field decouples from the vector field. The one-loop spinor self energy is computed using operator regularization, a technique which respects the three dimensional character of the antisymmetric tensor $\epsilon_{\alpha \beta \gamma}$. This method is used to evaluate the vector self energy to two-loop order; it is found to vanish showing that the beta function is zero to two-loop order. The canonical structure of the model is examined using the Dirac constraint formalism. 


\section{Introduction}

It has been pointed out $[1,2]$ that a suitable gauge invariant action for a massive vector field in three dimensions is

$$
S=\int d^{3} x\left[-\frac{1}{4}\left(\partial_{\mu} A_{\nu}-\partial_{\nu} A_{\mu}\right)^{2}-\frac{\mu}{2} \epsilon^{\mu \nu \lambda} A_{\mu} \partial_{\nu} A_{\lambda}+\bar{\psi}(i \not \partial-e \not A-m) \psi\right]
$$

The gauge coupling $e$ has dimension $[\text { mass }]^{1 / 2}$ indicating that the theory is super renormalizable; this is borne out by the structure of the vector propagator in a covariant gauge

$$
\begin{aligned}
& D_{\mu \nu}(p)=\frac{-i}{p^{2}-\mu^{2}+i \epsilon}\left[g_{\mu \nu}-\frac{p_{\mu} p_{\nu}}{p^{2}}-i \mu \epsilon_{\mu \nu \alpha} \frac{p^{\alpha}}{p^{2}}\right]-i a \frac{p_{\mu} p_{\nu}}{p^{4}} \\
& =\frac{-i}{p^{2}-\mu^{2}+i \epsilon}\left[g_{\mu \nu}-\frac{p_{\mu} p_{\nu}}{p^{2}}-i \epsilon_{\mu \nu \alpha} \frac{p^{\alpha}}{\mu}\right]-i\left(\frac{-i}{p^{2}+i \epsilon}\right)\left(-i \epsilon_{\mu \nu \alpha} \frac{p^{\alpha}}{\mu}\right)-i a \frac{p_{\mu} p_{\nu}}{p^{4}}
\end{aligned}
$$

where $a$ is a gauge parameter.

The Chern-Simons action on its own

$$
S=\int d^{3} x \epsilon^{\mu \nu \lambda} A_{\mu} \partial_{\nu} A_{\lambda}
$$

has itself been suggested as a suitable action for three dimensional vector field [3]; the nonAbelian extension has been extensively examined [4].

In this paper, we consider a model defined by the action

$$
\begin{gathered}
S=\int d^{3} x\left\{\frac{1}{2}\left[\epsilon^{\mu \alpha \nu} A_{\mu} \partial_{\alpha} A_{\nu}+\mu\left(A_{\mu}+\partial_{\mu} \phi\right)^{2}\right]\right. \\
+\bar{\psi}[\not p+e \not A-m] \psi\} \\
\left(\partial=i p, g_{\mu \nu}=(++-), \epsilon_{012}=1, \gamma_{\mu} \gamma_{\nu}=-g_{\mu \nu}+i \epsilon_{\mu \nu \lambda} \gamma^{\lambda}\right) .
\end{gathered}
$$

The kinetic part of the action for the vector field is now the Chern-Simons action (3); the part proportional to $\mu$ is a Stuekelberg mass term [5]. This interaction has been considered 
before in [6] where it was generated by considering a Chern-Simons plus Higgs action in the limit where the radial component of the Higgs field decoupled.

In the next section we will discuss the quantization of this model. The renormalization of the model will be discussed in section three and it will be shown by explicit calculation that to two-loop order, there is no renormalization of either the wave function $A_{\mu}$ or the mass parameter $\mu$. This entails using operator regularization [11], a technique which circumvents the necessity of trying to define the tensor $\epsilon_{\alpha \beta \gamma}$ outside of three dimensions. The method is first illustrated by computing the spinor two-point function to one-loop order. In section four the canonical structure of (4) is analyzed. A short discussion of the non-Abelian generalization of (4) is in a concluding section.

\section{Quantization of the Model}

The model defined by (4) possesses the $U(1)$ gauge invariance

$$
\begin{gathered}
A_{\mu} \rightarrow A_{\mu}+\partial_{\mu} \Lambda \\
\phi \rightarrow \phi-\Lambda \\
\psi \rightarrow e^{-i e \Lambda} \psi .
\end{gathered}
$$

In order to quantize this model, we add to the action the gauge fixing term

$$
S_{g f}=\int d^{3} x \frac{1}{2 a \mu}\left(\partial \cdot A+a \mu^{2} \phi\right)^{2}
$$

where $a$ is an arbitrary gauge parameter. With this choice, the fields $A_{\mu}$ and $\phi$ decouple and the free action for the vector field $A_{\mu}$ is just

$$
\begin{aligned}
S_{A} & =\int d^{3} x \frac{1}{2}\left\{\epsilon^{\mu \alpha \nu} A_{\mu} \partial_{\alpha} A_{\nu}+\mu A^{2}+\frac{1}{a \mu}(\partial \cdot A)^{2}\right\} \\
& \equiv \int d^{3} x \frac{1}{2} A_{\mu} M^{\mu \nu} A_{\nu} .
\end{aligned}
$$


It is easy to show that the inverse of the operator $M^{\mu \nu}$ defined in (7) is

$$
\begin{gathered}
\left(M^{-1}\right)_{\mu \nu}=\frac{1}{\partial^{2}-\mu^{2}} \epsilon_{\mu \alpha \nu} \partial^{\alpha}-\frac{\mu}{\partial^{2}-\mu^{2}} g_{\mu \nu} \\
+\frac{\mu(1-a)}{\left(\partial^{2}-\mu^{2}\right)\left(\partial^{2}-a \mu^{2}\right)} \partial_{\mu} \partial_{\nu} .
\end{gathered}
$$

From (8) it is apparent that the propagator for the field $A_{\mu}$ has a pole when its momentum $p$ satisfies the equation $p^{2}+\mu^{2}=0$, indicating that the vector field has a mass $\mu$. In contrast to the propagator of $(2 \mathrm{~b})$ there is no long range interaction in (8). Furthermore, the propagator behaves in leading order like $1 / p$ for large momentum, as is expected since the Chern-Simons action contains but one derivative. This is consistent with the model being renormalizable since we are in three dimensions. (We note in this context that the gauge coupling $e$ is now dimensionless.) If the field $\phi$ is set equal to zero in (4), then gauge invariance is lost and the propagator is the $a \rightarrow \infty$ limit of (8), which renders the theory unrenormalizable. This $\phi \rightarrow 0$ limit of (4) and the action of (1) have been considered in refs. [12] and [13]; indeed it was shown in [13] that the dynamical content of these two models is the same when the vector fields are free fields. Once the vector fields are coupled then the interactions of (4) and (1) are distinct in the $\phi=0$ gauge; the former interaction is non-renormalizable while the latter is renormalizable.

The bilinear part of the action for the scalar field $\phi$ has an inverse

$$
M^{-1}=\frac{1}{-\partial^{2}+a \mu^{2}} \frac{1}{\mu}
$$

This scalar field, however, is not coupled to the spinor $\psi$. If instead of (4) we consider the interaction

$$
S_{I}=\int d^{3} x \bar{\psi}\left(e_{1} \not \mathcal{A}-e_{2} \not \partial \phi\right) \psi
$$

with the gauge transformation $\psi \rightarrow e^{-i\left(e_{1}+e_{2}\right) \Lambda} \psi$, then $\phi$ does interact. The derivative coupling of $\phi$ with $\psi$ in (10) renders this interaction unrenormalizable and hence we restrict ourselves to the case $e_{2}=0$. 
The Faddeev-Popov ghost associated with the gauge fixing of (6) leads to a ghost propagator proportional to $1 /\left(-\partial^{2}+a \mu^{2}\right)$; however the ghost decouples from the remaining fields as the gauge condition is linear in the fields and the gauge transformation (5) is Abelian.

We now consider the renormalization of our model.

\section{Renormalization}

In order to compute radiative corrections in the model defined by (4), we must regulate ultraviolet divergences which arise in a way that is consistent with the three dimensional character of the tensor $\epsilon_{\alpha \beta \gamma}$. A variety of techniques, including dimensional regularization, a form of Pauli-Villars and the addition of a regulating Maxwell term to the action [2,710] have been employed. Perhaps the most straightforward approach is to use operator regularization [11], a symmetry preserving procedure in which no divergences ever appear explicitly and no regulating parameter is inserted into the original action, thereby leaving $\epsilon_{\alpha \beta \gamma}$ unambiguously defined. It has been employed in non-Abelian Chern-Simons theory $[14,15,16]$ to one and two loop order.

It is evident from naive power-counting arguments that ultraviolet divergences in the theory arise in two- and three-point Green's functions. The photon two-point Green's function in principal can generate divergences proportional to $A^{\mu} \epsilon_{\mu \rho \nu} \partial^{\rho} A^{\nu}$ and $\mu A^{\mu} A_{\mu}$; the former can be removed by renormalizing the photon wave function while the latter actually cannot arise because gauge invariance can be easily shown to imply that, as in four dimensional quantum electrodynamics (QED), radiative corrections to the two-point function must be transverse. The spinor two-point function is responsible for infinities proportional to $\bar{\psi} \not \phi \psi$ and $m \bar{\psi} \psi$; a spinor wave function and mass renormalization respectively can be used to eliminate these divergences. The only other divergent Green's function that can occur is the vertex function $\bar{\psi} A \psi$; a renormalization of the coupling constant $e$ eliminates this infinity. Since the form of the gauge transformations of (5) are identical to those in QED, the same arguments 
based on Ward identities [23] can be used to show that the wave function renormalization of the photon is in fact entirely responsible for the coupling constant renormalization. We consequently compute the divergent contribution to the photon two-point function.

Normally dimension regularization is the most convenient tool for handling divergences in gauge theories. However in the model of (4), the intrinsically three dimensional tensor $\epsilon_{\alpha \beta \gamma}$ occurs explicitly making it difficult to implement this technique. Operator regularization [11] is more suited to this theory since no regulating parameter is ever inserted into the initial Lagrangian leaving $\epsilon_{\alpha \beta \gamma}$ well defined at every stage of the calculation.

Background field quantization [24] is used in conjunction with operator regularization. The generating functional to a given order in the loop expansion is then written in closed form, and the logarithm of operators (at one-loop order) and the inverse of operators (beyond one-loop order) are then regulated; the initial Lagrangian is never altered such as by inserting a regulating Pauli-Villars mass or by analytically continuing the number of space-time dimensions. We illustrate this technique by first using it to compute the spinor self energy to one-loop order.

In order to compute this contribution to the effective action, we first provide $\psi$ with a background piece $\chi$. The one-loop generating functional is then given by the superdeterminant

$$
\Gamma^{(1)}(\chi, \bar{\chi})=\operatorname{sdet}^{-\frac{1}{2}}\left(\begin{array}{ccc}
i \epsilon_{\mu \alpha \nu} p^{\alpha}+\mu g_{\mu \nu}+\frac{1}{\mu} p_{\mu} p_{\nu} & -e\left(\gamma_{\mu} \chi\right)^{T} & e\left(\bar{\chi} \gamma_{\mu}\right) \\
e\left(\gamma_{\nu} \chi\right) & 0 & \not p-m \\
-e\left(\bar{\chi} \gamma_{\nu}\right)^{T} & -\not p^{T}+m & 0
\end{array}\right)
$$

in the gauge in which $a=1$.

We now want to extract the $(\bar{\chi} \chi)$ contribution to $\Gamma^{(1)}(\chi, \bar{\chi})$. To do that, we first multiply (11) by the constant matrix

$$
X=\operatorname{sdet}^{-\frac{1}{2}}\left(\begin{array}{ccc}
-i \epsilon_{\mu \rho \nu} p^{\rho}+\mu g_{\mu \nu} & 0 & 0 \\
0 & 0 & \not p^{T}+m \\
0 & -\not p-m & 0
\end{array}\right)
$$


so that

$$
\Gamma^{(1)}(\chi, \bar{\chi})=\operatorname{sdet}^{-\frac{1}{2}}\left(\begin{array}{ccc}
\left(p^{2}+\mu^{2}\right) g_{\mu \nu} & -e\left(\bar{\chi} \gamma_{\mu}\right)(\not p+m) & -e\left(\gamma_{\mu} \chi\right)^{T}\left(\not p^{T}+m\right) \\
e\left(\gamma^{\kappa} \chi\right)\left(-i \epsilon_{\kappa \lambda \nu} p^{\lambda}+\mu g_{\kappa \nu}\right) & p^{2}+m^{2} & 0 \\
-e\left(\bar{\chi} \gamma_{\kappa}\right)^{T}\left(-i \epsilon_{\kappa \lambda \nu} p^{\lambda}+\mu g_{\kappa \nu}\right) & 0 & p^{2}+m^{2}
\end{array}\right)
$$

In operator regularization [11], we first write

$$
\text { sdet } H=\exp s t r \ln H
$$

and then regulate $\ln H$

$$
\ln H=-\left.\frac{d}{d s}\right|_{0} H^{-s}
$$

so that

$$
\Gamma^{(1)}=\exp \frac{1}{2} \zeta^{\prime}(0)
$$

where [14]

$$
\zeta(s)=\frac{1}{\Gamma(s)} \operatorname{str} \int_{0}^{\infty} \operatorname{dit}(i t)^{s-1} \exp -(i H t) .
$$

To extract the contribution to $\zeta(s)$ which is bilinear in $(\bar{\chi} \chi)$ we employ either the Schwinger expansion [18,11] or employ the quantum mechanical path integral [19]. Upon identifying $H$ with the operator appearing in (13), we find that

$$
\begin{aligned}
\zeta_{\bar{\chi} \chi}(s) & =\frac{i e^{2}}{(4 \pi)^{\frac{3}{2}}} \frac{2}{\Gamma(s)} \int d^{3} p \int_{0}^{1} d u \bar{\chi}(p)\left\{\frac{3 \Gamma\left(s-\frac{1}{2}\right)}{\left[u(1-u) p^{2}+u \mu^{2}+(1-u) m^{2}\right]^{s-\frac{1}{2}}}\right. \\
& \left.+\frac{\Gamma\left(s+\frac{1}{2}\right)\left[\not p(u \mu+2(1-u) m)+3 \mu m-2 u(1-u) p^{2}\right]}{\left[u(1-u) p^{2}+u \mu^{2}+(1-u) m^{2}\right]^{s+\frac{1}{2}}}\right\} \chi(-p) .
\end{aligned}
$$

As is expected, since this is a three dimensional theory, no dependence on logarithms of $p^{2}$ or the masses arises in $\zeta_{\bar{\chi} \chi}^{\prime}(0)$; such logarithms can appear only beyond one-loop order. Similarly, divergences appear in renormalizable three dimensional scalar models only beyond one-loop order [20] when using dimensional regularization. 
Having seen how radiative corrections cannot result in contributions to the renormalization group functions until at least two-loop order when one employs operator regularization in an odd number of dimensions, we will turn our attention to the vacuum polarization at two-loop order. As has been discussed above, this will determine the two-loop contribution to the renormalization of the coupling constant and hence will fix the beta function to this order in perturbation theory.

The two-loop generating functional in the presence of external vector field $A_{\mu}$ can be shown to be

$$
\begin{gathered}
\Gamma^{(2)}[A]=\frac{-i e^{2}}{2} \int d x d y<x\left|\left(-i \epsilon_{\mu \rho \nu} p^{\rho}+\mu g_{\mu \nu}\right)\left(p^{2}+\mu^{2}\right)^{-1}\right| y> \\
\operatorname{Tr}\left\{\gamma^{\mu}<x\left|(\not p+e \not A)\left[(p+e A)^{2}-\frac{e}{2} \epsilon_{\alpha \beta \lambda} F^{\alpha \beta} \gamma^{\lambda}\right]^{-1}\right| y>\right. \\
\left.\gamma^{\nu}<y\left|(\not p+e \not A)\left[(p+e A)^{2}-\frac{e}{2} \epsilon_{\gamma \delta \sigma} F^{\gamma \delta} \gamma^{\sigma}\right]^{-1}\right| x>\right\} .
\end{gathered}
$$

This follows from (4) and (6) upon setting $a=1, m=0$.

Just as (15) can be used to regulate the logarithm of an operator, the inverse of an operator can be regulated using

$$
H^{-1}=\left.\frac{d^{n}}{d s^{n}}\right|_{0} \frac{s^{n}}{n !} H^{-s-1} . \quad(n=1,2 \cdots)
$$

This allows us regulate $\Gamma^{(2)}[A]$ in (19) in the following way

$$
\begin{aligned}
& \Gamma^{(2)}[A]=\left.\frac{d}{d s}\right|_{0} s\left(\frac{-i e^{2}}{2}\right) \int_{0}^{\infty} \frac{d i t_{1} d i t_{2} d i t_{3}}{\Gamma^{3}(s+1)}\left(i t_{1} i t_{2} i t_{3}\right)^{s} \\
& \int d x d y<x\left|\left(-i \epsilon_{\mu \rho \nu} p^{\rho}+\mu g_{\mu \nu}\right) \exp -i\left[p^{2}+\mu^{2}\right] t_{1}\right| y> \\
& \operatorname{Tr}\left\{\gamma^{\mu}<x\left|(\not p+e \not A) \exp -i\left[(p+e A)^{2}-\frac{e}{2} \epsilon_{\alpha \beta \lambda} F^{\alpha \beta} \gamma^{\lambda}\right] t_{2}\right| y>\right. \\
&\left.\gamma^{\nu}<y\left|(\not p+e \not A) \exp -i\left[(p+e A)^{2}-\frac{e}{2} \epsilon_{\gamma \delta \sigma} F^{\gamma \delta} \gamma^{\sigma}\right] t_{3}\right| x>\right\} .
\end{aligned}
$$

It is possible to neglect "regulated forms of zero" discussed in [26-28,30] in (21) since in three dimensions one-loop subgraphs are not divergent. This also means that we can choose the parameter $n$ in (20) to be equal to one. 
Since we are interested in only the logarithmic dependent pieces of the two-point function, it is much easier to employ the DeWitt expansion [25] rather than compute the full two-point function from the Schwinger expansion [18]. The utility of this procedure at two-loop order has previously been illustrated $[16,26,27]$. In this technique we make use of the following expansion

$$
<x\left|e^{-i\left[(p+A)^{2}+f\right] t}\right| y>=\frac{e^{i(x-y)^{2} / 4 t}}{(4 \pi i t)^{D / 2}} \sum_{n=0}^{\infty} a_{n}(x, y)(i t)^{n}
$$

in $D$ dimensions. All dependence on $A_{\mu}$ and $f$ is contained in the coefficients $a_{n}(x, y)$. As has been argued, in order to determine the photon wave function renormalization, the terms in the effective action that we need to consider are bilinear in $A_{\mu}$ and contain at most one derivative of $A_{\mu}$; consequently the coefficients $a_{n}(x, y)$ need to be determined only to second order in $A_{\mu}$ and first order in $\partial_{\lambda} A_{\mu}$. This can be easily done using the techniques of [29]; we find that

$$
\begin{aligned}
&<x\left|e^{-i\left[(p+e A)^{2}-\frac{e}{2} \epsilon_{\mu \nu \lambda} F^{\mu \nu} \gamma^{\lambda}\right] t}\right| y> \\
&=\frac{e^{i \Delta^{2} / 4 t}}{(4 \pi i t)^{D / 2}}\left\{\left[1-i e \Delta \cdot A-\frac{e^{2}}{2}(\Delta \cdot A)^{2}\right]\right. \\
&\left.+i t[1-i e \Delta \cdot A]\left(\frac{e}{2} \epsilon_{\mu \nu \lambda} F^{\mu \nu} \gamma^{\lambda}\right)\right\}
\end{aligned}
$$

so that to the required order

$$
\begin{aligned}
<x\left|(\not p+e \not A) e^{-i\left[(p+e A)^{2}-\frac{e}{2} \epsilon_{\mu \nu \lambda} F^{\mu \nu} \gamma^{\lambda}\right] t}\right| y> \\
=\frac{e^{i \Delta^{2} / 4 t}}{(4 \pi i t)^{3 / 2}}\left\{\frac{\not \Delta}{2 t}+e\left[-\frac{i \not \Delta \Delta \cdot A}{2 t}+\frac{i \not \Delta}{4} \epsilon_{\mu \nu \lambda} F^{\mu \nu} \gamma^{\lambda}-\frac{\gamma_{\mu} \Delta_{\lambda}}{2} F^{\mu \lambda}\right]\right. \\
\left.+e^{2}\left[-\frac{\not \Delta(\Delta \cdot A)^{2}}{4 t}+\frac{\not}{4} \Delta \cdot A \epsilon_{\mu \nu \lambda} F^{\mu \nu} \gamma^{\lambda}+\frac{i}{2} \gamma_{\mu} \Delta_{\lambda} F^{\mu \lambda} \Delta \cdot A\right]\right\}
\end{aligned}
$$

(Here $\Delta=x-y$ and all fields are evaluated at $z=\frac{x+y}{2}$.)

Upon substitution of (24) into (21), the two-point function, to first order in derivatives of the external wave function, is

$$
\Gamma_{A A}^{(2)}=\left.\frac{-i e^{4}}{2} \frac{d}{d s}\right|_{0} s \int d^{3} z d^{3} \Delta \int_{0}^{\infty} \frac{d^{2} t_{1} d i t_{2} d_{i t}}{\Gamma^{3}(s+1)} \frac{\left(i t_{1} i t_{2} i t_{3}\right)^{s-3 / 2}}{(4 \pi i)^{9 / 2}}
$$




$$
\begin{gathered}
e^{-i \mu^{2} t_{1}} e^{\frac{i \Delta^{2}}{4}\left(\frac{1}{t_{1}}+\frac{1}{t_{2}}+\frac{1}{t_{3}}\right)}\left(-i \epsilon_{\mu \rho \nu} \frac{\Delta^{\rho}}{2 t_{1}}+\mu g_{\mu \nu}\right) \\
\operatorname{tr}\left\{\gamma^{\mu}\left(\frac{\not}{2 t_{2}}\right) \gamma^{\nu}\left(\frac{\not \Delta(\Delta \cdot A)^{2}}{4 t_{3}}+\frac{\not}{4} \Delta \cdot A \epsilon_{\alpha \beta \lambda} F^{\alpha \beta} \gamma^{\lambda}+\frac{i}{2} \gamma_{\alpha} \Delta_{\lambda} F^{\alpha \lambda} \Delta \cdot A\right)\right. \\
+\gamma^{\mu}\left(-\frac{\not \Delta(\Delta \cdot A)^{2}}{4 t_{2}}+\frac{\not \Delta}{4} \Delta \cdot A \epsilon_{\alpha \beta \lambda} F^{\alpha \beta} \gamma^{\lambda}+\frac{i}{2} \gamma_{\alpha} \Delta_{\lambda} F^{\alpha \lambda} \Delta \cdot A\right) \gamma^{\nu}\left(-\frac{\Delta}{2 t_{3}}\right) \\
+\gamma^{\mu}\left(-\frac{i \not \Delta(\Delta \cdot A)}{2 t_{2}}+\frac{i \not \Delta}{4} \epsilon_{\alpha \beta \lambda} F^{\alpha \beta} \gamma^{\lambda}-\frac{\gamma_{\alpha} \Delta_{\lambda}}{2} F^{\alpha \lambda}\right) \gamma^{\nu}\left(-\frac{i \not \Delta(\Delta \cdot A)}{2 t_{3}}\right. \\
\left.\left.-\frac{i \not \Delta}{4} \epsilon_{\gamma \delta \sigma} F^{\gamma \delta} \gamma^{\sigma}+\frac{\gamma_{\beta} \Delta_{\sigma}}{2} F^{\beta \sigma}\right)\right\} .
\end{gathered}
$$

In (25) we can immediately discard terms with an odd number of factors of $\Delta_{\mu}$. Remarkably, the remaining terms proportional to $\mu^{2} A^{2}$ and $\epsilon_{\mu \lambda \nu} A^{\mu} \partial^{\lambda} A^{\nu}$ automatically cancel, eliminating the need to evaluate any integrals explicitly or to compute any traces of gamma matrices. (All integrals could, in fact, be determined using the techniques of $[26,28,30]$.) The fact that we obtain a vanishing result even prior to having to compute potentially divergent integrals indicates that the use of operator regularization is superfluous; Pauli-Villars regularization could also have been used to obtain this result (although it would have been computationally more difficult).

We consequently see that no renormalization of either the vector wave function $A_{\mu}$ or the mass parameter $\mu$ occurs to two-loop order, so that the beta function and anomalous mass dimension vanish to this order. The vanishing of the two-loop beta function is in accordance with the results of [31].

\section{Canonical Formalism}

We first note that the equations of motion associated with the Lagrangian (4) are

$$
\epsilon^{\mu \lambda \nu} \partial_{\lambda} A_{\nu}+\mu\left(\partial^{\mu} \phi+A^{\mu}\right)+j^{\mu}=0
$$

and

$$
\partial_{\mu}\left(\partial^{\mu} \phi+A^{\mu}\right)=0
$$


upon varying $A^{\mu}$ and $\phi$ respectively. The field $A^{\mu}$ has been coupled to a classical source $j^{\mu}$. If we act on (26a) with the operator $\epsilon_{\alpha \beta \mu} \partial^{\beta}$, then we obtain

$$
-\partial_{\alpha}\left(\partial \cdot A+\mu^{2} \phi\right)+\left(\partial^{2}-\mu^{2}\right) A_{\alpha}=\mu j_{\alpha}-\epsilon_{\alpha \beta \gamma} \partial^{\beta} j^{\gamma}
$$

which upon applying the gauge condition

$$
\partial \cdot A+\mu^{2} \phi=0
$$

shows that $A_{\alpha}$ is indeed a field with mass $\mu$. Furthermore, if we combine (26a) and (26b), we see that $j^{\mu}$ must be conserved (viz $\left.\partial \cdot j=0\right)$.

We now show how these results can be interpreted in the context of the canonical formalism for constrained systems as developed by Dirac [21]. A complete formulation of the quantization of this model has already been presented by Boyanovsky [6]. Our quantization procedure differs from that of [6] in that we replace, in the Lagrangian, $2 A_{\mu} \partial^{\mu} \phi$ by the equivalent symmetrized expression $A^{\mu} \partial_{\mu} \phi-\phi \partial_{\mu} A^{\mu}$. This leads to different expressions for $\pi_{0}$ and $\pi_{\phi}$. Furthurmore, in [6] the constraints associated with the momenta $\pi_{i}$ are immediately classified as being second class; the first class constraints are discussed only after the two second class constraints are used to define the appropriate Dirac brackets (i.e. after the corresponding variables $A_{i}$ are identified as a canonical pair). In contrast, we determine the class of the constraints in the system by considering all four constraints simultaneously. The physical content of the two approaches is identical.

We begin by determining the canonical momenta,

$$
\begin{aligned}
& \pi_{\phi}=\frac{\partial \mathcal{L}}{\partial\left(\partial^{0} \phi\right)}=\mu\left(\partial_{0} \phi+\frac{1}{2} A_{0}\right) \\
& \pi_{0}=\frac{\partial \mathcal{L}}{\partial\left(\partial^{0} A^{0}\right)}=\frac{\mu}{2} \phi \\
& \pi_{i}=\frac{\partial \mathcal{L}}{\partial\left(\partial^{0} A^{i}\right)}=\frac{1}{2} \epsilon_{i j} A_{j} \quad\left(\epsilon_{0 i j} \equiv \epsilon_{i j}\right),
\end{aligned}
$$


from which we derive the Hamiltonian

$$
\begin{aligned}
\mathcal{H}= & \frac{\mu}{2} A_{0}^{2}-\frac{1}{2 \mu}\left(\pi_{\phi}-\frac{\mu}{2} A_{0}\right)^{2}+A_{0} \epsilon_{i j} \partial_{i} A_{j} \\
& -\frac{\mu}{2}\left(\partial_{i} \phi+A_{i}\right)^{2}-j_{i} A_{i}+j_{0} A_{0} .
\end{aligned}
$$

It is evident that (29b) and (29c) are primary constraint equations. By computing the Poisson bracket $\left\{\pi_{0}-\frac{\mu}{2} \phi, \mathcal{H}\right\}$ we find the secondary constraint

$$
\pi_{\phi}+\epsilon_{i j} \partial_{i} A_{j}+j_{0}+\frac{\mu}{2} A_{0}=0
$$

(If the $\mu=0$ component of (26a) is satisfied, then (29a) and (31) are compatible.)

Unlike the corresponding constraint of ref. [6], equation (31) cannot be identified with the generator of gauge transformations in our approach, as it does not commute with the constraint of (29c). The first class constraints in our model, then, are (29b) and a linear combination of (31) and (29c),

$$
\pi_{\phi}+\partial_{i} \pi_{i}+\frac{1}{2} \epsilon_{i j} \partial_{i} A_{j}+j_{0}+\frac{\mu}{2} A_{0}=0
$$

It is easily shown that the Poisson bracket of (32) with $\mathcal{H}$

is zero and hence there are no tertiary constraints; no other linear combination of constraints $(29 \mathrm{c})$ and $(31)$ has this property. The Gauss law constraint $\partial_{i} E_{i}=0$ in ordinary electrodynamics is analogous to (32), as (32) generates the gauge transformation

$$
\begin{aligned}
& \phi \rightarrow \phi+\Lambda \\
& A_{i} \rightarrow A_{i}-\partial_{i} \Lambda .
\end{aligned}
$$

Gauge conditions compatible with (29a) and (32) are

$$
\begin{aligned}
A_{0} & =0 \\
\partial_{i} A_{i}+\mu^{2} \phi & =0 ;
\end{aligned}
$$


these are analogous to the usual Coulomb gauge conditions in electrodynamics.

The remaining two linear combinations of constraints in (29c) and (31) constitute a pair of second class constraints. We thus see that in our model there are two second class constraints, two first class constraints and two gauge conditions, thereby reducing the number of degrees of freedom from eight to two: the (single) transverse polarization of the vector and its canonical conjugate.

We note that the action of (4) is equivalent to a Freedman-Townsend [32] type of action

$$
\begin{aligned}
S=\int d^{3} x & \frac{\mu}{2} \epsilon^{\mu \alpha \beta} \phi_{\mu} F_{\alpha \beta}(A+V)+\frac{\mu^{2}}{2} V_{\mu} V^{\mu} \\
+ & \left.\frac{m}{2} \epsilon^{\mu \alpha \beta} A_{\mu} F_{\alpha \beta}(A)\right\}
\end{aligned}
$$

upon applying the equation of motion to the field $\phi_{\mu}$. The quantization of the action in (35) is treated in [33] using the Batalin-Fradkin-Vilkovisky procedure [34].

\section{Discussion}

We have demonstrated that a renormalizable massive Abelian vector theory exists in three dimensions. Regrettably, it does not appear possible to extend the model of (4) to a nonAbelian gauge theory. The replacement of the Abelian kinetic term with a non-Abelian Chern-Simons action [4]

$$
S_{C S}=\int d^{3} x \frac{1}{2} \epsilon^{\mu \nu \lambda}\left(A_{\mu}^{a} \partial_{\nu} A_{\lambda}^{a}-\frac{1}{3} f^{a b c} A_{\mu}^{a} A_{\nu}^{b} A_{\lambda}^{c}\right)
$$

and the Stuekelberg mass term with a Kunimasa-Goto action [22]

$$
S_{K G}=\int d^{3} x \mu\left(A_{\mu}^{a}-i\left(U^{-1} \partial_{\mu} U\right)^{a}\right)^{2}
$$

results in a gauge invariant action. However, decoupling the field $U$ from $A_{\mu}$ through a judicious choice of gauge condition (i.e. finding the non-Abelian generalization of (6)) does not appear to be feasible. Consequently it is apparently not possible to find a renormalizable 
model of a massive non-Abelian gauge field in three dimensions without invoking the Higgs mechanism.

The model of (4) is quite similar to one considered in ref. [35]. The authors of [35] have looked at the infrared limit of a vector theory in $2+1$ dimensions defined by a Chern-Simons and Proca mass term with a view of applying the model to anyon physics.

\section{Acknowledgments}

We would like to thank the Natural Science and Engineering Research Council of Canada for financial support, and to Prof. A.J. Niemi for bringing ref. [32] to our attention.

\section{References}

[1] J. Schonfeld, Nucl. Phys. B185, 157 (1981).

[2] S. Deser, R. Jackiw and S. Templeton, Ann. of Phys. 140, 372 (1982).

[3] C.R. Hagen, Ann. of Phys. 157, 342 (1984).

[4] E. Witten, Comm. Math. Phys. 121, 351 (1989).

[5] E.C.G. Stuekelberg, Helv. Phys. Acta 11, 225 (1938).

[6] D. Boyanovsky, Phys. Rev. D42, 1179 (1990).

[7] C.P. Martin, Phys. Lett. B241, 513 (1990). M. Asorey and F. Falceto, Phys. Lett. B241, 31 (1990). G. Giavarini, C.P. Martin and F. Ruiz Ruiz, Nucl. Phys. B381, 222 (1992).

[8] L. Alvarez-Gaumé, J.M.F. Labastida and A.V. Ramallo, Nucl. Phys. B334, 103 (1990).

[9] E. Guadagnini, M. Martellini and M. Mintchev, Phys. Lett. B227, 111 (1989). 
[10] W. Chen, G.W. Semenoff and Y.-S. Wu, in Physics, Geometry and Topology (NATO ASI Series, Plenum Press, New York) ed. H.C. Lee.

[11] D.G.C. McKeon and T.N. Sherry, Phys. Rev. D35, 3854 (1987).

[12] P.K.Townsend, K. Pilch and P. von Nieuwenhuizen, Phys. Lett. 136B, 38 (1984).

[13] S. Deser and R. Jackiw, Phys. Lett. 139B, 371 (1984).

[14] D.G.C. McKeon, Can. J. Phys. 68, 1291 (1990).

[15] D. Birmingham, H.T. Cho, R. Kantowski and M. Rakowski, Phys. Rev. D42, 3476 (1990).

[16] D.G.C. McKeon and S.K. Wong, Int. J. Mod. Phys. A (to be published).

[17] S. Hawking, Commun. Math. Phys. 55, 133 (1977).

[18] J. Schwinger, Phys. Rev. 82, 664 (1951).

[19] D.G.C. McKeon, Ann. of Phys. 224, 139 (1993).

[20] R.D. Pisarski, Phys. Rev. Lett. 48, 574 (1982).

[21] P.A.M. Dirac, Can. J. Math. 2, 129 (1950); 3, 1 (1951).

[22] T. Kunimasa and T. Goto, Prog. Theor. Phys. 37, 452 (1967).

[23] J.D. Bjorken and S.D. Drell, Relativistic Quantum Mechanics, McGraw-Hill (New York 1964).

[24] B.S. DeWitt, Phys. Rev. 162, 1195, 1239 (1967).

L.F. Abbott, Nucl. Phys. B185, 189 (1981). 
[25] B. DeWitt, Dynamical Theory of Groups and Fields, Gordon and Breach (New York 1965).

R.T. Seeley, Amer. Math. Soc. 10, 228 (1967).

P.B. Gilkey, J. Diff. Geom. 10, 601 (1975).

[26] L. Culumovic, D.G.C. McKeon and T.N. Sherry, Ann. of Phys. (N.Y.) 197, 94 (1989).

[27] L. Culumovic and D.G.C. McKeon, Can. J. Phys. 68, 1166 (1990).

[28] L. Culumovic, M. Leblanc, R.B. Mann, D.G.C. McKeon and T.N. Sherry, Phys. Rev. D41, 514 (1990).

[29] L. Culumovic and D.G.C. McKeon, Phys. Rev. D38, 3831 (1988).

F.A. Dilkes and D.G.C. McKeon, UWO report (1995) unpublished.

[30] M. Leblanc, R.B. Mann, D.G.C. McKeon and T.N. Sherry, Nucl. Phys. B349, 494 (1991).

[31] L.V. Avdeev, G.V. Grigoryev and D.I. Kazakov, Nucl. Phys. B382, 561 (1992).

A.W. Kapustin and P.I. Pronin, Phys. Lett. B318, 465 (1993).

[32] D.Z. Freedman and P.K. Townsend, Nucl. Phys. B177, 282 (1981).

[33] D.G.C. McKeon, Int. J. Mod. Phys. A, 7, 2005 (1992).

[34] I.A. Batalin and G.A. Vilkovisky, Phys. Rev. D28, 2567 (1983).

E.S. Fradkin and G.A.Vilkovisky, Phys. Lett. 55B, 224 (1975).

[35] A.J. Niemi and V.V. Sreedhar, Phys. Lett. B336, 381 (1994). 